\title{
Influence of Treatment Complexity on Adherence and Incidence of Blips in HIV/HCV Coinfected Patients
}

\author{
Elena Calvo-Cidoncha, BPharm; Javier González-Bueno, BPharm; \\ Carmen Victoria Almeida-González, MS; and Ramón Morillo-Verdugo, BPharm
}

\begin{abstract}
BACKGROUND: The addition of antihepatitis $C$ therapy to highly active antiretroviral treatment (HAART) in human immunodeficiency virus (HIV)/hepatitis $\mathrm{C}$ virus (HCV) coinfected patients leads to an increase in the treatment complexity that may result in decreased adherence. Blips, defined as intermittent episodes of detectable low-level HIV viremia, may be an indication of poor adherence to HAART.
\end{abstract}

OBJECTIVES: To (a) determine the influence of adding anti-HCV therapy to HAART on complexity index, adherence, and incidence of blips and (b) determine complexity index and adherence in patient subgroups based on anti-HCV therapy.

METHODS: We conducted a prospective 2-center observational study. HIV/HCV coinfected patients under antiretroviral treatment who started anti-HCV bi-therapy or triple therapy between January 2011 and December 2013 were included. Patients were excluded if they were virologically uncontrolled (HIV viral load $>50$ copies RNA/mL) or if they had changed antiretroviral treatment in the 6 months prior to the introduction of antiHCV therapy. Data were collected before and after the addition of anti-HCV therapy to HAART. The main variables were complexity index, incidence of blips, and adherence. The complexity index was based on a score that utilized the number of pills per day, dosing schedule, dosage form, and any specific instructions linked to use of the drug. Blips were defined as a detectable HIV-RNA level ( $>50$ copies/mL but no more than 1,000 copies $/ \mathrm{mL}$ ) occurring between 2 negative assays. Medication adherence was assessed using electronic pharmacy refill records. The threshold for optimal adherence was defined at $95 \%$ and above. Differences in the variables collected were assessed before and after the addition of anti-HCV therapy to HAART.

RESULTS: A total of 66 patients were included in the study. Based on the complexity index, the median value before and after the addition of antiHCV therapy to HAART was 4.2 (interquartile range $[I Q R]=3.5-5.5$ ) and $11.5($ IQR $=10.4-13.4)$, respectively. The median difference between both complexity indices was $6.9(95 \% \mathrm{Cl}=6.9-7, P<0.001)$. After introducing the anti-HCV therapy into HAART, the number of adherent patients decreased from $50(75.8 \%)$ to $45(68.2 \%, P>0.05)$, and $12(18.2 \%)$ patients presented blips $(P<0.001)$. Subgroup analysis based on anti-HCV therapy showed that patients on boceprevir or telaprevir therapy had a higher complexity index, 16.8 (IQR=6.0-18.4), compared with patients on bi-therapy antiHCV, 11.3 (IQR = 10.3-12). The median difference was $6.0(95 \% \mathrm{Cl}=5.0-7.2$, $P<0.001)$. The number of adherent patients decreased only in patients on bi-therapy from $42(79.2 \%)$ to $37(69.8 \%, P>0.05)$.

CONCLUSIONS: Adding anti-HCV therapy to antiretroviral treatment significantly increases treatment complexity and the incidence of blips. The introduction of anti-HCV therapy is also associated with a decrease in the number of adherent patients. The regimen complexity calculation may be useful for identifying patients who need more care from health care professionals or are at risk for failure to comply with treatment regimens.

J Manag Care Pharm. 2015;21(2):153-57

Copyright $\odot 2015$, Academy of Managed Care Pharmacy. All rights reserved.

\section{What is already known about this subject}

Adherence to antiretroviral treatment is an important predictive factor in treatment outcome.

Human immunodeficiency virus (HIV)/hepatitis C virus (HCV) coinfection increases the risk of liver disease progression and worsens clinical outcomes.

\section{What this study adds}

The addition of anti-HCV therapy to antiretroviral treatment leads to a significant increase in the complexity index associated with a decrease in the number of adherent patients.

The addition of anti-HCV therapy to antiretroviral treatment increases significantly the incidence of blips in virologically controlled patients.

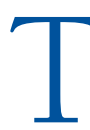
he natural history of the human immunodeficiency virus (HIV) has changed significantly since the appearance of highly active antiretroviral treatment (HAART) in 1996. ${ }^{1}$ Since then, the morbidity and mortality associated with the disease has drastically reduced, and patients' quality of life has increased. ${ }^{2}$

Currently, HIV infection is considered a chronic illness. However, adherence to HAART is an important predictive factor in treatment outcome. ${ }^{3,4}$ There are multiple reasons for failing to adhere; adverse events related to therapy and drug-drug interactions are some of the main causes of nonadherence..$^{5-7}$

Medication nonadherence has also been linked to medication regimen complexity. ${ }^{8,9}$ In 2011, the American Society of Health-System Pharmacists published a consensus document about optimal pharmacy practice models in hospitals and health systems. ${ }^{10}$ One of the specific points mentioned in this consensus was that pharmacist-provided drug therapy management should be prioritized using a patient medication complexity index. 
Medication count is not an adequate complexity measurement because it only addresses pill burden. Others factors may also contribute to complexity. A tool developed by Martin et al. in 2007 includes factors such as the number of medications, dosing schedules, administration methods, and special dosing instructions when determining the complexity of treatment. ${ }^{11}$ Weights are applied for each of these factors to produce an overall score representing the regimen's level of complexity.

Intermittent episodes of detectable low level viremia, referred to as blips, are observed frequently in HIV-infected patients who receive antiretroviral therapy. Although the etiology of transient viremia is poorly understood, many clinicians have hypothesized that poor adherence to the medication regimen is responsible for transient viremia. ${ }^{12}$ Others studies suggest that the phenomenon of blips originates from activation and expansion of latently infected cells and from the appearance and selection of new drug-resistant strains. ${ }^{13,14}$

Approximately $30 \%$ of HIV-infected patients are coinfected with hepatitis $\mathrm{C}$ virus (HCV). After the release of highly active antiretroviral therapy, liver disease has become the leading cause of morbidity and mortality in HIV patients. ${ }^{15}$ Until 2011, the standard therapy for the treatment of hepatitis $C$ was based on the combination therapy of pegylated interferon alfa- $2 a$ or alfa-2b and ribavirin administered for 24 or 48 weeks. ${ }^{16}$ However, the treatment of hepatitis $C$ is evolving at a rapid pace. In 2011, the first 2 protease inhibitors, telaprevir and boceprevir, were approved. More recently, several oral antiviral agents that inhibit various HCV proteins, such as sofosbuvir, simeprevir, and daclatasvir, have been developed. These new agents have caused a radical change in the prognosis of the disease. ${ }^{17,18}$ However, the addition of anti-HCV therapy to HAART leads to an increase in the treatment complexity, which may result in decreased adherence. ${ }^{19}$

To date, no study has evaluated the effect of introducing antiHCV therapy to HIV treatment on the control of HIV disease; therefore, the main objective of this study was to determine the influence of adding anti-HCV therapy to HAART on the complexity index, adherence, and incidence of blips. A second objective was to determine the complexity index and the adherence in patient subgroups based on their anti-HCV therapy.

\section{Methods}

We conducted a prospective 2-center observational study. HIV/HCV coinfected patients under antiretroviral treatment who started anti-HCV bi-therapy or triple therapy between January 2011 and December 2013 were included. Patients were excluded if they were virologically uncontrolled (HIV viral load $>50$ copies RNA per milliliter $[/ \mathrm{mL}]$ ) or if they had changed antiretroviral treatment in the 6 months prior to the introduction of anti-HCV therapy. The latter criterion was to reduce potential bias in measurement of adherence. The collected data included age, gender, race, antiretroviral treatment, anti-HCV therapy, weeks on anti-HCV therapy, and incidence of blips. Additionally, we determined adherence and the complexity index of the treatment before and after the addition of antiHCV therapy to HAART.

Blips were defined as a detectable HIV-RNA level (> 50 copies/mL but no more than 1,000 copies $/ \mathrm{mL}$ ) occurring between 2 negative assays. Calculation of complexity index was performed through a web application ${ }^{20}$ based on an adaptation of the score created by Martin et al. ${ }^{11}$ This score utilized the number of pills per day, dosing schedule, dosage form, and any specific instructions related to drug use. According to the weights added to these factors, a single number that represents the overall complexity index is calculated. This score has been previously validated, and it is applicable to children and adults with HIV disease and HIV/HCV coinfected patients. ${ }^{11,21}$

Because the complexity of anti-HCV therapy varies among treatment options, we took into account the highest value possible in statistical analysis. This value corresponds with the addition of a direct-acting antiviral to bi-therapy.

Medication adherence was assessed using electronic pharmacy refill records. The proportion of days covered was calculated based on the following formula: [(pills dispensed/ pills prescribed per day)/days between refills] $\times 100$. The threshold for optimal adherence was defined at $95 \%$ and above. Adherence before the addition of anti-HCV therapy to HAART was assessed for 6 months. After anti-HCV therapy introduction into HAART, adherence was assessed from the initial prescription of anti-HCV therapy until its date of discontinuation. The remaining data were collected through outpatient electronic medical records and by reviewing the medical history of each patient.

Quantitative variables are expressed as mean and standard deviation or as median and percentile P25 and P75 in case of a skewed distribution. Qualitative variables are expressed as percentages (\%). In order to determine the significance of the differences between the variables collected before and after the addition of anti-HCV therapy to HAART, we ran the following statistical analysis: when data were consistent with a normal distribution, a t-test for related samples was used to compare 2 means of quantitative variables. Otherwise, a nonparametric Wilcoxon test was performed. The Mann-Whitney U test was also performed on the subgroup analysis. The confidence interval (CI) established to determine differences between mean or median was $95 \%$. The McNemar's test was applied to analyze changes in dichotomous variables. Data analysis was carried out using the statistical package SPSS 20.0 for Windows (SPSS Inc., Chicago, IL).

\section{Results}

Sixty-six patients were included in the study, 36 from a tertiary hospital and 30 from a secondary hospital. Demographics and 


\begin{tabular}{l|c}
\hline \multicolumn{1}{c|}{ TABLE 1) } & $\begin{array}{l}\text { Demographic Characteristics } \\
\text { of Study Patients }\end{array}$ \\
\hline Variable & Frequency \\
\hline Sex: male/female (\% male) & \multicolumn{2}{c}{$57 / 9(86.4)$} \\
\hline Age (years) & \multicolumn{2}{c}{$47 \pm 4.8$} \\
\hline Caucasian race, $n(\%)$ & $66 \quad(100.0)$ \\
\hline
\end{tabular}

pharmacotherapy characteristics are detailed in Table 1 and Table 2, respectively.

Considering the antiretroviral drug classes, the most common regimens were those including a combination of a nonnucleoside and a nucleoside reverse transcriptase inhibitor (36.4\%). The most prescribed regimen consisted of efavirenz/ emtricitabine/tenofovir (16.6\%). None of the patients had to change antiretroviral therapy during the study period. With respect to the anti-HCV treatment, bi-therapy was most commonly used. In patients receiving triple therapy for HCV, telaprevir was preferred over boceprevir. The median duration of the anti-HCV treatment was 45.6 (interquartile range $[I Q R]=20.4-49.1)$ weeks.

Based on the complexity index, the median value before and after the addition of anti-HCV therapy to HAART was 4.2 $(\mathrm{IQR}=3.5-5.5)$ and $11.5(\mathrm{IQR}=10.4-13.4)$, respectively. The median difference between both complexity indices was 6.9 (95\% CI $6.9-7.0, P<0.001$ ). Sixteen $(24.2 \%)$ patients presented transient intermittent viremia after the addition of anti-HCV therapy to HAART. Twelve patients (18.2\%) fit our definition of blips. The incidence of blips was statistically significant $(P<0.001)$. Fifty $(75.8 \%)$ patients were considered adherent to HAART. After introducing the anti-HCV therapy into HAART, the number of adherent patients decreased to 45 (68.2\%). This decline was not statistically significant.

There were statistically significant differences depending on the anti-HCV treatment regimen for complexity index. The median complexity index value in patients with bi-therapy was $11.3(\mathrm{IQR}=10.3-12)$ compared with $16.8(\mathrm{IQR}=16.0-18.4)$ in patients on triple-therapy anti-HCV. The median difference was 6.0 (95\% CI = 5.0-7.2, P<0.001).

Table 3 shows data on adherence for patients treated with bi-therapy or triple therapy. After the addition of anti-HCV therapy to HAART, the number of adherent patients decreased only in patients on bi-therapy, but it was not modified in patients on triple therapy. The decrease in the number of adherent patients after adding the bi-therapy anti-HCV was not statistically significant.

\section{Discussion}

Our study shows that the addition of anti-HCV therapy to HAART leads to a significant increase in the complexity index and incidence of blips. Results of subgroup analysis based on
TABLE 2 Pharmacotherapy of Study Patients

\begin{tabular}{l|rc}
\hline \multicolumn{2}{c}{ Antiretroviral Treatment } \\
\hline Combination of Antiretroviral Drugs & \multicolumn{2}{c}{ Frequency, $\mathbf{n}(\%)$} \\
\hline NRTI + NNRTI & 24 & $(36.4)$ \\
\hline NRTI + PI & 15 & $(22.7)$ \\
\hline NRTI + INI & 12 & $(18.2)$ \\
\hline Monotherapy & 10 & $(15.2)$ \\
\hline Others & 5 & $(7.6)$ \\
\hline \multicolumn{2}{c}{ Antihepatitis C Virus Treatment } & \\
\hline Hepatitis C Virus Therapy & Frequency, $\mathbf{n}(\%)$ \\
\hline Peginterferon $\alpha 2 \mathrm{a} / \alpha 2 \mathrm{~b}+$ ribavirin & 53 & $(80.3)$ \\
\hline PegInterferon $\alpha 2 \mathrm{a} / \alpha 2 \mathrm{~b}+$ ribavirin + telaprevir & 11 & $(16.7)$ \\
\hline PegInterferon $\alpha 2 \mathrm{a} / \alpha 2 \mathrm{~b}+$ ribavirin + boceprevir & 2 & $(3.0)$ \\
\hline
\end{tabular}

INI= integrase inhibitor; NNRTI = non-nucleoside reverse transcriptase inhibitor; $N R T I=$ nucleoside reverse transcriptase inhibitor; $P I=$ protease inhibitor .

anti-HCV therapy showed that patients on boceprevir or telaprevir therapy have a higher complexity index compared with patients on bi-therapy anti-HCV.

The number of adherent patients decreased after introducing the anti-HCV therapy into HAART. This decline was not statistically significant; however, it could be clinically significant. Nonadherence to therapy is closely associated with incomplete viral suppression and disease progression and is thought to be a risk factor for the development of drug resistance. ${ }^{22}$

To date there is no evidence of the influence of treatment complexity on adherence of HIV/HCV coinfected patients. However, there is literature in HIV monoinfected patients. Stone et al. (2001) examined the complexity of antiretroviral regimens by assessing administration instructions and dosing frequency. ${ }^{23}$ Their results indicated that patients whose regimens included a more complex medication were more likely to become nonadherent. Similar conclusions can be drawn from the work of Paterson et al. (2000), which reports a significantly greater adherence to twice-daily dosing versus 3 times daily. ${ }^{24}$ In contrast, regimen complexity was not a significant predictor of adherence in the study of Gao et al. (2000). ${ }^{25}$ None of the previous studies have used a reliable and valid method to quantify the complexity of HAART in HIV patients.

Our results do not show a significant decrease in the number of adherent patients. There are a number of reasons for this. Despite the growing burden of HCV/HIV coinfected patients, the limited duration of anti-HCV therapy might promote adherence. In addition, patients receiving anti-HCV therapy, especially patients on boceprevir or telaprevir, experienced an intensive monitoring, which included pharmacologic management of treatment-related adverse effects and education of patients. ${ }^{26,27}$ As a result, adherence may be improved.

To date, there are no studies of a relationship between treatment complexity and incidence of blips. Many studies 
Influence of Treatment Complexity on Adherence and Incidence of Blips in HIV/HCV Coinfected Patients

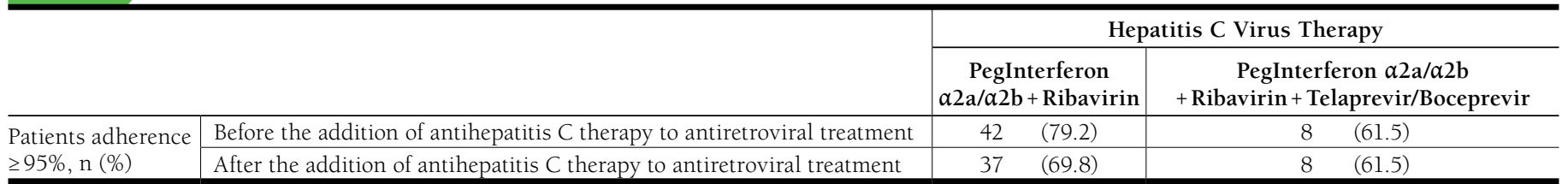

have evaluated the association between blips and adherence; however, the natural history and clinical significance of blips remain unclear. ${ }^{12,28,29}$ As discussed by Fung et al. (2012), a contributing factor to the diverse outcomes of the various studies on viral blips is that there has been no consensus on the definition of viral blips. ${ }^{30}$ The regimen complexity index should be used in daily practice in order to identify HIV/HCV coinfected patients at a higher risk of having transient intermittent viremias and who need a more intense adherence monitoring.

\section{Limitations and Implications for Further Research}

The main limitation in this study is that the complexity index only incorporates antiretroviral medications and anti-HCV treatment. HIV/HCV coinfected patients often take prophylactic therapy for opportunistic infections or other concomitant drugs to counteract the adverse effects induced by HAART or anti-HCV therapy. The complexity conferred by concomitant drugs is not considered in the complexity index analysed in this study. Thus, an issue for future work could be to assess the influence of treatment complexity on adherence and incidence of blips, considering any additional medications that the patient may be taking along with the HIV/HCV treatments. With the advent of direct-acting anti-HCV drugs, a marked increase in the rate of virologic response is observed in coinfected patients. ${ }^{31}$ So, another important area for future research is to determine whether suppression of HCV replication could be associated with a higher efficacy of antiretroviral therapy.

Another limitation of this study is the use of pharmacy refill records for measuring HAART adherence because indirect methods could lead to an overestimation. To overcome this possible bias, patients were considered adherent when their drug uptake was higher than 95\%. Nevertheless, according to current literature, a $90 \%$ threshold would be enough to achieve desirable outcomes. ${ }^{32}$

In addition to complexity index, there are numerous other techniques currently available to researchers that may in the future be incorporated into clinical routine. These include the analysis of human and viral genetic determinants of disease evolution, detailed analyses of immune recovery and reserve, pharmacogenetic determinants of treatment response, and toxicity. In the future, these approaches may provide highly individualized disease management. ${ }^{33}$

\section{Conclusions}

Adding anti-HCV therapy to antiretroviral treatment significantly increases the treatment complexity and incidence of blips. The introduction of anti-HCV therapy is also associated with a tendency towards a decrease in the number of adherent patients. The regimen complexity calculation may be useful for identifying patients who need more care from health care professionals or are at risk for failure to comply with their treatment regimens.

\section{Authors}

ELENA CALVO-CIDONCHA, BPharm, is a Pharmacy Resident, Pharmacy Department, and RAMÓN MORILLO-VERDUGO, BPharm, is Specialist, Hospital Pharmacy, Nuestra Señora de Valme Hospital, Seville, Spain. JAVIER GONZÁLEZ-BUENO, BPharm, is a Pharmacy Resident, Pharmacy Department, Virgen del Rocio Hospital, and CARMEN VICTORIA ALMEIDA-GONZÁLEZ, MS, is Professor, University of Seville, and Statistician, Research Unit, Valme University Hospital, Seville, Spain.

AUTHOR CORRESPONDENCE: Elena Calvo-Cidoncha, BPharm, Pharmacy Department, Nuestra Señora de Valme Hospital, Av. Bellavista, s/n, 41014. Seville, Spain. Tel.: +34.654355751;

E-mail: elena.calci@gmail.com.

\section{DISCLOSURES}

The authors have no conflicts of interest to declare.

Study concept and design were contributed by Calvo-Cidoncha and González-Bueno, who also collected the data. Data interpretation was perfomed by Almeida-González and Calvo-Cidoncha. The manuscript was written by Calvo-Cidoncha, González-Bueno, and Morillo-Verdugo, and revised by all the authors equally.

\section{REFERENCES}

1. Crane HM, Van Rompaey SE, Kitahata MM. Initiating highly active antirretroviral therapy with newer protease inhibitor is associated with better survival compared to first generation protease inhibitors or nevirapine. AIDS Patient Care STDS. 2007;21(12):920-29.

2. Sterne JA, Hernan MA, Ledergerber B, et al. Long-term effectiveness of potent antiretroviral therapy in preventing AIDS and death: a prospective cohort study. Lancet. 2005;366 (9483):378-84.

3. Kitahata MM, Reed SD, Dillingham PW, et al. Pharmacy-based assessment of adherence to HAART predicts virological and immunologic treatment response and clinical progression to AIDS and death. Int J STD AIDS. 2004;15(12):803-10. 
4. Bangsberg DR, Perry S, Charlebois ED, et al. Non-adherence to highly active antiretroviral therapy predicts progression to AIDS. AIDS. 2001;15(9):1181-83.

5. Mok S, Minson Q. Drug-related problems in hospitalized patients with HIV infection. Am J Health Syst Pharm. 2008;65(1):55-59.

6. Elzi L, Marzolini C, Furrer H, et al. Treatment modification in human immunodeficiency virus-infected individuals starting combination antiretroviral therapy between 2005 and 2008. Arch Intern Med. 2010;170(1):57-65.

7. Johnston SS, Juday T, Seekins D, Espindle D, Chu BC. Association between prescription cost sharing and adherence to initial combination antiretroviral therapy in commercially insured antiretroviral-naive patients with HIV. J Manag Care Pharm. 2012;18(2):129-45. Available at: http://www.amcp. org/WorkArea/DownloadAsset.aspx?id=14756.

8. Stone VE, Jordan J, Tolson J, et al. Perspectives on adherence and simplicity for HIV-infected patients on antiretroviral therapy: self-report of the relative importance of multiple attributes of highly active antiretroviral therapy (HAART) regimens in predicting adherence. J Acquir Immune Defic Syndr. 2004;36(3):808-16. Available at: http://journals.lww.com/jaids/pages/ articleviewer. aspx? year $=2004 \&$ \&ssue $=07010 \&$ article $=00007 \&$ type $=$ abstract. Accessed December 22, 2014.

9. Yuan Y, L'italien G, Mukherjee J, et al. Determinants of discontinuation of initial highly active antiretroviral therapy regimens in a US HIV-infected patient cohort. HIV Med. 2006;7(3):156-62. Available at: http://onlinelibrary. wiley.com/doi/10.1111/j.1468-1293.2006.00355.x/pdf. Accessed December 22, 2014

10. Anonymous. The consensus of the Pharmacy Practice Model Summit. Am J Health Syst Pharm. 2011;68(12):1148-52. Available at: http://www.ajhp. org/content/68/12/1148.full.pdf+html. Accessed December 22, 2014.

11. Martin S, Wolters PL, Calabrese SK, et al. The Antiretroviral Regimen Complexity Index. A novel method of quantifying regimen complexity. J Acquir Immune Defic Syndr. 2007;45(5):535-44.

12. Podsadecki TJ, Vrijens BC, Tousset EP, Rode RA, Hanna GJ. Decreased adherence to antirretroviral therapy observed prior to transient human immunodeficiency virus type 1 viremia. J Infec Dis. 2007;196(12):1773-78. Available at: http://jid.oxfordjournals.org/content/196/12/1773.full.pdf+html. Accessed December 22, 2014.

13. Jones LE, Perelson AS. Transient viremia, plasma viral load, and reservoir replenishment in HIV-infected patients on antiretroviral therapy. J Acquir Immune Defic Syndr. 2007;45(5):483-93. Available at: http://www. ncbi.nlm.nih.gov/pmc/articles/PMC2584971/. Accessed December 22, 2014.

14. Hermankova M, Ray SC, Ruff C, et al. HIV-1 drug resistance profiles in children and adults with viral load of $<50$ copies $/ \mathrm{ml}$ receiving combination therapy. JAMA. 2001;286(2):196-207. Available at: http://jama.jamanetwork. com/article. aspx?articleid=193999. Accessed December 22, 2014.

15. Bichoupan K, Dieterich DT. Hepatitis C in HIV-infected patients: impact of direct-acting antivirals. Drugs. 2014;74(9):951-61.

16. McHutchison JG, Lawitx EJ, Shiffman ML, et al. Peginterferon alfa-2b or alfa-2a with ribavirin for treatment of hepatitis C infection. N Eng J Med. 2009;361(6):580-93. Available at: http://www.nejm.org/doi/pdf/10.1056/ NEJMoa0808010. Accessed December 22, 2014.

17. Ahn J, Flamm SL. Frontiers in the treatment of hepatitis $C$ virus infection. Gastroenterol Hepatol (NY). 2014;10(2):90-100.Available at: http://www ncbi.nlm.nih.gov/pmc/articles/PMC4011382/. Accessed December 22, 2014.

18. Thomas DL. Advances in the treatment of hepatitis $C$ virus infection. Top Antivir Med. 2012;20(1):5-10. Available at: http://www.iasusa.org/sites/ default/files/tam/20-1-5.pdf. Accessed December 22, 2014.
19. Gaglio PJ, Moss N, McGaw C, Reinus J. Direct-acting antiviral therapy for hepatitis C: attitudes regarding future use. Dig Dis Sci. 2011;56(5):150915. Available at: http://www.ncbi.nlm.nih.gov/pmc/articles/PMC3082020/ pdf/10620_2011_Article_1604.pdf. Accessed December 22, 2014.

20. Consulta de atención farmacéutica. Patologías víricas. Índice de Complejidad. Available at: http://www.farmaciavalmecpv.com/consulta/ actividad/indice-de-complejidad/. Accessed December 22, 2014.

21. Galán RJ, Cidoncha EC, Martin MF, Rodriguez CC, Almeida CV, Verdugo RM. Antiviral regimen complexity index as an independent predictor of sustained virologic response in patients with chronic hepatitis C. J Manag Care Pharm. 2013;19(6):448-53. Available at: http://www.amcp.org/ WorkArea/DownloadAsset.aspx?id=16751.

22. Fielden SJ, Rusch ML, Yip B, et al. Nonadherence increases the risk of hospitalization among HIV-infected antiretroviral naive patients started on HAART. J Int Assoc Physicians AIDS Care (Chic). 2008;7(5):238-44.

23. Stone VE, Hogan JW, Schuman P, et al. Antiretroviral regimen complexity, self-reported adherence, and HIV patients' understanding of their regimens: survey of women in the HER study. J Acquir Immune Defic Syndr. 2001;28(2):124-31.

24. Paterson DL, Swindells S, Mohr J, et al. Adherence to protease inhibitor therapy and outcomes in patients with HIV infection. Ann Intern Med. 2000;133(1):21-30.

25. Gao X, Nau DP, Rosenbluth SA, et al. The relationship of disease severity, health beliefs and medication adherence among HIV patients. AIDS Care. 2000;12(4):387-98.

26. Spooner, LM. The critical need for pharmacist involvement in the management of patients with hepatitis C. J Manag Care Pharm. 2009;15(2):15153. Available at: http://www.amcp.org/data/jmcp/151-153.pdf.

27. Mariño EL, Alvarez-Rubio L, Miró S, et al. Pharmacist intervention in treatment of patients with genotype 1 chronic hepatitis C. J Manag Care Pharm. 2009;15(2):147-50. Available at: http://www.amcp.org/data/jmcp/147150.pdf.

28. Lee PK, Kieffer TL, Siliciano RF, Nettles RE. HIV-1 viral load blips are of limited clinical significance. J Antimicrob Chemother. 2006;57(5):803-05. Available at: http://jac.oxfordjournals.org/content/57/5/803.full.pdf+html. Accessed December 22, 2014.

29. Miller LG, Golin CE, Liu H, et al. No evidence of an association between transient HIV viremia ("blips") and lower adherence to the antiretroviral medication regimen. J Infect Dis. 2004;189(8):1487-96.

30. Fung IC, Gambhir M, van Sighem A, de Wolf F, Garnett GP. The clinical interpretation of viral blips in HIV patients receiving antiviral treatment: are we ready to infer poor adherence? J Acquir Immune Defic Syndr. 2012;60(1):5-11.

31. Bani-Sadr F, Loko MA, Pambrun E, et al. Correlates of HIV sustained viral suppression in HIV/hepatitis $C$ virus coinfected patients: possible role of the hepatitis C virus sustained viral response. AIDS. 2014;28(8):1155-60.

32. Bangsberg DR. Less than $95 \%$ adherence to nonnucleoside reversetranscriptase inhibitor therapy can lead to viral suppression. Clin Infect Dis. 2006;43(7):939-41. Available at: http://cid.oxfordjournals.org/content/43/7/939.full.pdf+html. Accessed December 22, 2014.

33. Telenti A. New developments in laboratory monitoring of HIV-1 infection. Clin Microbiol Infect. 2002;8(3):137-43. Available at: http://onlinelibrary. wiley.com/doi/10.1046/j.1469-0691.2002.00408.x/pdf. Accessed December 22, 2014. 